\title{
Meyer, Michel. 2008. Principia Rhetorica. Une théorie générale de l'argumentation (Paris : Fayard)
}

\section{Marc Angenot}

\section{(2) OpenEdition}

\section{Journals}

Édition électronique

URL : http://journals.openedition.org/aad/749

DOI : $10.4000 /$ aad. 749

ISSN : 1565-8961

Éditeur

Université de Tel-Aviv

\section{Référence électronique}

Marc Angenot, «Meyer, Michel. 2008. Principia Rhetorica. Une théorie générale de l'argumentation (Paris : Fayard) », Argumentation et Analyse du Discours [En ligne], 3 | 2009, mis en ligne le 15 octobre 2009, consulté le 21 septembre 2020. URL : http://journals.openedition.org/aad/749; DOI : https://doi.org/ 10.4000/aad.749

\section{Ce document a été généré automatiquement le 21 septembre 2020}

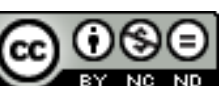

Argumentation \& analyse du discours est mis à disposition selon les termes de la licence Creative Commons Attribution - Pas d'Utilisation Commerciale - Pas de Modification 4.0 International. 


\title{
Meyer, Michel. 2008. Principia Rhetorica. Une théorie générale de l'argumentation (Paris : Fayard)
}

\author{
Marc Angenot
}

\section{RÉFÉRENCE}

Meyer, Michel. 2008. Principia Rhetorica. Une théorie générale de l'argumentation (Paris :

Fayard), 327 p.

1 De Logique, langage et argumentation, en 1982, à Qu'est-ce que l'argumentation? (2005), l'œuvre magistrale de Michel Meyer comporte, nul ne l'ignore, plusieurs ouvrages déjà, étalés sur plus de vingt ans, qui traitent sous diverses perspectives et selon divers aspects de questions de rhétorique argumentative.

2 Les Principia qui viennent de paraître, pourrait-on penser, viennent alors former la synthèse de cette forte et complexe réflexion philosophique dispersée dans les livres antérieurs. Certes, ils forment cette synthèse, qui était attendue, de la rhétorique intégrée à la philosophie du questionnement, à la problématologie formulée par le philosophe bruxellois, mais les Principia Rhetorica sont autre chose encore, de plus ambitieux et de plus englobant. À cet égard, un peu curieusement, le sous-titre de ce livre est trompeur: "une théorie générale de l'argumentation »? Non, c'est trop restreint et trop « modeste ». L'ambition de Meyer, ambition qui structure tout le livre, est de prendre à bras le corps et d'inscrire dans un ensemble philosophiquement cohérent les pratiques discursives, dans toute leur diversité, qui permettent «la négociation de la distance entre des individus à propos d'une question donnée » pratiques qui vont de l'argumentation apodictique, de la coopération en vue d'accord, à la disputatio, à l'éristique des «conflits", à la figuralité poétique, à l'expression énigmatique ou hermétique. Le livre part de cette définition et aboutit à un vaste 
questionnement typologique, développé à grands traits, "Comment négocie-t-on la distance entre individus? » (227 et ss.)

3 La définition que je viens de rappeler, voulue contraire de l'optimisme normatif des la tradition rhétorique, est neutre quant au résultat de la «négociation»: ce peut être persuader, convaincre, clarifier, mais aussi d'aventure, accroître la distance entre les entreparleurs, obscurcir les choses et rendre plus «problématique » (au sens banal de ce mot) l'échange langagier.

4 Autrement dit, Meyer, en partant de cette définition désormais bien connue, déjà creusée dans ses livres antérieurs, définition large qui rompt avec la conception restreinte traditionnelle de la rhétorique comme pragmatique oratoire persuasive, prétend réintégrer en un tout ce que l'histoire des réflexions sur le discours depuis Aristote (car c'est bien sur cette durée de vingt-quatre siècles que Meyer aborde la question !) avait progressivement séparé : la rhétorique de l'argumentation, isolée de la rhétorique des figures et des tropes depuis avant l'âge classique, mais aussi de la poétique et de l'herméneutique.

5 C'est en effet un des mérites, pas assez perçu et souligné par la critique, de Meyer d'intégrer la réflexion sur la littérature et la poésie (on a même droit, ici et là, à l'esquisse d'une théorie de l'art dramatique) à celle sur l'analyse des discours « exotériques » et du raisonnement public. En fait, de nos jours, il est le seul à faire une telle chose et il perturbe ici encore des cloisonnements commodes et en tout cas inquestionnés. À cet égard je rappelle son Langage et littérature (1992) qui est, trop mal connu à mon sens, un livre décisif (voir aussi sa Petite métaphysique de la différence. 2000). Certaines des perspectives développées dans ce livre se trouvent au reste creusée dans les Principia.

6 Bien entendu qui connaît l'œuvre de Meyer ne sera pas surpris de la visée totalisatrice qui est ici développée dans toute son ampleur et, en quelque sorte, dans sa systématicité délibérée qu'illustrent tableaux et graphiques. Il s'agit pour Meyer, à partir de sa philosophie du questionnement - montrée par lui comme le « remède » aux apories et stérilités de la pensée propositionnaliste occidentale - de réconcilier en un tout co-intelligible les disjecta membra des pensées sur le discours, et réciproquement il s'agit pour lui, très expressément et fort légitimement, de montrer que seule la problématologie permet de penser globalement, c'est-à-dire en un tout dont les divers cas de figure s'éclairent réciproquement, ce qu'une tradition séculaire a vainement et contre-productivement compartimenté. Je ne reviendrai pas sur la problématologie même dont on peut trouver une excellente présentation sommaire mais d'une grande clarté dans le petit livre d'Angèle Kremer-Marietti, Michel Meyer et la problématologie (2008. Bruxelles : Éd. de l'Université).

7 Toutes les notions fondamentales, «qu'est-ce qu'un argument? ", «qu'est ce qu'un lieu?», «quels sont les types d'auditoires?», se trouvent alors reformulées et systématiquement rediscutées. Seule la problématologie, pose Meyer, permet ainsi de penser le lien, jadis défait et qui demeure à ce jour non-élucidé, entre la rhétorique des figures et des tropes et la rhétorique des arguments. Cette proposition heuristique au cœur du livre mérite réflexion. Dans les Principia, on (re-)trouve en tout cas une rhétorique tout d'un tenant, sans couture et sans division (85 et ss.). Le chapitre sur la « loi d'unité » des deux champs, - l'un qui argumente en implicitant et présupposant (par rapport à une "question qui se pose ») et l'autre qui invite à inférer du figural au 
littéral/intentionnel, du dit au vouloir-dire. Meyer développe, une fois encore à contrecourant, l'idée féconde d'une " rationalité du figuratif » (129).

C'est ici, dans l'ampleur de la synthèse recherchée, l'ambition de ce livre voulu fondamental - livre qui n'est nullement, on l'aura bien compris, un traité de rhétorique avec ses listes de figures, avec ses schemata et sa topique, mais la construction axiomatique des principes qui doivent fonder, à son sens, toute réflexion sur l'échange langagier entre questionnement et déchiffrement.

9 Meyer a raison de le formuler en titre d'un de ses chapitres, c'est bien d'une «nouvelle vision de la rhétorique " qu'il est question. Quand il est discuté dans les Principia de telles figures et tropes, de tels procédés et de genres et sous-genres, ce n'est jamais que pour illustrer la séquence de principes fondateurs qui sont posés et articulés entre eux de chapitre en chapitre. Le livre de Meyer ne quitte pas ce niveau fondamental et il bâtit, avec une sorte de jubilation pédagogique fonceuse, un enchainement systématique qui se veut aussi une synthèse - éblouissante d'érudition maîtrisée - des réflexions rhétoriques séculaires d'Aristote à l'Âge classique, à Perelman, à Toulmin et à nos jours. À la fin du $20^{\mathrm{e}}$ siècle, "La rhétorique renaît lorsque les systèmes idéologiques s'effondrent ", suggère justement Meyer (De la métaphysique à la rhétorique. Essais à la mémoire de Chaïm Perelman, 1986: 7). «La volonté de soumettre les affaires humaines à une eschatologie scientifique a échoué », il reste aux (post-) modernes, la recherche négociée d'une «entente » et d'un consensus (B. Buffon. La parole persuasive 2002: 73). Le grand regain rhétorique a été ainsi le contemporain du Second désenchantement, celui des religions séculières ou politiques ; de l'éloignement de l'univoque, de l'apodictique, des vérités définitives, scientifiques ou dogmatiques.

Le début des Principia consiste notamment en une discussion de diverses approches de jadis et naguère du fait global que Meyer nomme "rhétorique", - approches divergentes qui privilégient toutes un aspect et abordent le fait global sous un angle donné, celui de l'ethos, du pathos, ou du logos, de l'orateur, du langage, de l'auditoire... Ethos, logos et pathos sont au contraire confrontés par Meyer comme les «trois sources des réponses » au questionnement (151 et ss.).

11 Sans doute Meyer rend-il hommage, sincère à n'en pas douter, à Chaïm Perelman, son maître et le point de départ de tout ce qui s'est fait en rhétorique francophone depuis un demi-siècle, mais on peut dire néanmoins, et Meyer n'en fait pas mystère, que les Principia sont aux antipodes et forment en réalité une réfutation intégrale des quatre traits axiomatiques ou quatre partis pris du Traité de l'argumentation : la prédominance du logos, la mise à l'écart du pathos et le droit/l'argumentation juridique comme modèles des pratiques oratoires, l'indifférence ipso facto à la situation d'échange langagier et donc au "traitement d'une différence » qui est au cœur de la réflexion de Meyer. (Meyer me semble plutôt proche à bien des égards de H.-G. Gadamer, mais il reproche à celui-ci de ne pas creuser assez le questionnement - 64, p. ex.).

12 J'ai qualifié de "pédagogie fonceuse » le style philosophique de Meyer qui ne s'attarde jamais, style d'idées qui n'est pas celui des prudences d'une pensée bien circonscrite, pédestre et auto-limitée, une pensée qui regimberait devant les vastes questions qui semblent se poser à mesure qu'on avance. Or, Meyer avance souvent à grandes enjambées et il lui arrive alors de régler en quelques lignes, suggestives sans doute, des problèmes pour lesquels il ne consent pas toujours à lever intégralement la perplexité du lecteur. Il en va ainsi de ce paradigme auquel il a recours fréquemment et dès le début (et qui fonde son idée de la littérature comme représentation de l'historicité- 
devenir entre identité et métaphoricité) / accélération de l'histoire / délitement des réponses naguères acquises et accroissement de la problématicité /, paradigme dont on perçoit intuitivement le potentiel, mais auquel on voudrait lui demander de s'attarder avec des illustrations plus fouillées (ou peut-être d'y consacrer tout un livre?): «l'histoire en s'accélérant métaphorise de plus en plus les vieilles réponses qui sont de moins en moins des réponses et de plus en plus des énigmes ».

Les Principia ne sont pas un livre facile, il ne servirait de rien de le cacher et le lecteur de ce compte rendu s'en doute - mais il en est peu d'aussi stimulant, d'aussi généreux intellectuellement, d'aussi utilement provocateur encore dans la mise en cause des conceptions reçues et dans le renouvellement des... questionnements. C'est un livre qui marque son époque.

\section{AUTEURS}

\section{MARC ANGENOT}

Titulaire de la Chaire James McGill d'Etude du Discours social, Université Mc Gill, Montréal 